\title{
Platelet-activating factor receptor activation promotes prostate cancer cell growth, invasion and metastasis via ERK1/2 pathway
}

\author{
WENBIN JI ${ }^{1}$, JIN CHEN $^{1}$, YUCHENG MI ${ }^{1}$, GUANLIANG WANG ${ }^{1}$, XINJIAN XU $^{1}$ and WEIZHEN WANG ${ }^{2}$ \\ Departments of ${ }^{1}$ Radiology, ${ }^{2}$ Neurology, Taizhou Hospital of Wenzhou Medical University, Taizhou, Zhejiang 317000, P.R. China
}

Received January 4, 2016; Accepted February 14, 2016

DOI: $10.3892 /$ ijo.2016.3519

\begin{abstract}
Platelet-activating factor (PAF) and its receptor (PAFR), have been reported to participate in many cellular processes of cancer. However, little is known about their function in prostate cancer. In the present study, we found that PAFR was overexpressed in prostate cancer cells. PAF stimulation dose-dependently promoted the invasion, migration and growth of prostate cancer cells in vitro, while knockdown of PAFR inhibited the effect of PAF on prostate cancer cells. We further found that PAFR promoted prostate cancer cell growth and metastasis in vivo. Moreover, we found that PAFR activation increased MMP-3 expression and decreased E-cadherin expression of prostate cancer cells in vitro and in vivo. Finally, we found that PAFR time-dependently induced activation of ERK1/2, and ERK1/2 pathway contributed to PAFR-mediated prostate cancer cell invasion, migration and growth. Together, our findings demonstrate that PAFR can activate ERK1/2 pathway, and subsequently increase MMP-3 expression and decrease E-cadherin expression, which finally promote prostate cancer cell growth, invasion and metastasis. Thus, PAFR may act as a potential target for therapeutic use of prostate cancer.
\end{abstract}

\section{Introduction}

Prostate cancer is one of the most common types of cancer worldwide, and acts as a leading cause of cancer-related death in men (1). While early prostate cancer can be cured, patients with advanced prostate cancer often suffered from invasion and metastasis, which leads to death of prostate cancer patients (2). Therefore, discovering novel molecular biomarkers associated with prostate cancer progression, especially those regulating the invasion and metastasis, may provide potential molecular targets for the detection and therapy of prostate cancer.

Correspondence to: Dr Weizhen Wang, Department of Neurology, Taizhou Hospital of Wenzhou Medical University, 150 Ximen Road, Taizhou, Zhejiang 317000, P.R. China

E-mail: wangwzll@126.com

Key words: platelet-activating factor receptor, prostate cancer, invasion, metastasis, ERK1/2, growth
PAF is known as a phospholipid mediator of inflammation, and further studies proved that PAF plays a pivotal role in many diseases, including cancer $(3,4)$. It is reported that PAF promotes the migration and proliferation of breast cancer cells (5), and contributes to the invasiveness and motility of melanoma cells (6). PAF exerts its biological effects mainly via activating PAFR. As a member of G-protein coupled receptor (GPCR) family, PAFR has been found to promote the malignant development of esophageal squamous cell carcinoma via PI3K/AKT pathway (7), and enhance the growth of ovarian cancer cells through cooperating with EGFR (8). However, it is reported that elevated PAFR expression is significantly associated with smaller tumor size, absence of lymph node and organ metastasis and low tumor histopathological stage in gastric adenocarcinoma (9). In prostate cancer, the function of PAFR remains elusive.

Given that PAFR may act as contrasting effect on the progression of different cancers, the present study aimed to investigate the effect of PAF and PAFR interaction in the progression of prostate cancer cells. We unexpectedly found that activation of PAFR by PAF stimulated the growth, invasion and metastasis of prostate cancer cells via the ERK1/2 pathway.

\section{Materials and methods}

Cell lines and reagents. All cell lines were purchased from the American Type Culture Collection (ATCC; Manassas, VA, USA). Human prostate cancer cell lines LNCap, PC-3, PC-3M and DU-145 were maintained in RPIM-1640 medium containing $10 \%$ fetal bovine serum (FBS) in $5 \% \mathrm{CO}_{2}$ atmosphere at $37^{\circ} \mathrm{C}$. Normal prostate cell line RPWE-1 was maintained in K-SFM medium containing $10 \%$ FBS in 5\% $\mathrm{CO}_{2}$ atmosphere at $37^{\circ} \mathrm{C}$. ERK1/2 special inhibitor U0126, PAF and DMSO were purchased from Sigma-Aldrich (St. Louis, MO, USA). Antibodies against PAFR, E-cadherin, ERK1/2 and $\beta$-actin were obtained from Santa Cruz Biotechnology (Santa Cruz, CA, USA). Antibody against phosho-ERK1/2 was obtained from Cell Signaling Technology (Danvers, MA, USA).

Cell transfection. A specific siRNA targeting PAFR (siPAFR) was purchased from GenePharma Co., Ltd., (Shanghai, China) with the sequence of 5'-CUGGGCGUCAUCACUUAUA-3' to transiently silence PAFR expression. A scramble siRNA was 
used as control siRNA (siCtrl). Cells were incubated with siPAFR or siCtrl for $36 \mathrm{~h}$ using the Lipofectamine 2000 transfection reagent (Invitrogen), according to the manufacturer's instruction. Knockdown efficiency was determined by western blotting, and the cells were subjected to the experiments described below.

Furthermore, to stably silence PAFR expression in DU-145 cells, a PAFR shRNA (shPAFR) was obtained from GenePharma, while a scrambled shRNA was used as control shRNA (shcontrol). Cells were incubated with shPAFR or shControl for $36 \mathrm{~h}$ using the Lipofectamine 2000 transfection reagent (Invitrogen), and the stable clones were selected by G418. Knockdown efficiency was detected by western blotting.

Western blotting. Total proteins from cell lines or transplanted tumor tissues of mice were isolated using RIPA buffer with protease inhibitor and phosphatase inhibitor. The concentration of protein was determined by BCA assay (Applygen Technologies, Inc., Beijing, Beijing, China). Then, equal amounts of protein were separated in $10 \%$ polyacrylamide gel and electrotransferred on PVDF membrane (Millipore Corp., Billerica, MA, USA). The membrane was probed with primary antibodies overnight, and then incubated with secondary antibodies for $1 \mathrm{~h}$. Next, the membrane was visualized by enhanced chemiluminescence kit (Applygen Technologies). The density of each band was analyzed with Quantity One software.

In vitro invasion assay and migration assay. In vitro invasion assay and migration assay were performed using Transwell chambers (Corning Costar, Corning, NY, USA). Briefly, cells at the density of $1 \times 10^{6}$ cells $/ \mathrm{ml}$ were plated in serum-free medium on upper chambers coated with Matrigel in invasion assay, whereas $600 \mu 1$ of 1640 medium supplemented with $20 \%$ FBS was added into the lower chambers. Sixteen hours later, cells passed through the membrane were stained with crystal violet, and the numbers of cells were counted under a light microscope in seven random fields. For in vitro migration assay, cells at the density of $1 \times 10^{6}$ cells $/ \mathrm{ml}$ were plated in serum-free medium on upper chambers without Matrigel, and cells were allowed to migrate for $16 \mathrm{~h}$. The migrated cells were stained with crystal violet, and the numbers of cells were counted under a light microscope in seven random fields.

Real-time PCR. Total RNA was isolated from cells using TRIzol reagent (Invitrogen), following the manufacturer's instruction. Then, reverse transcription PCR was carried out using Omniscript RT-PCR kit (Qiagen, Hilden, Germany). Next, real-time PCR was performed using the primers of E-cadherin (forward, 5'-CGAGAGCTACACGTTCACGG-3' and reverse, 5'-GGGTGTCGAGGGAAAAATAGG-3'; MMP-3 (forward, 5'-CTGGACTCCGACACTCTGGA-3' and reverse, 5'-CAGGAAAGGTTCTGAAGTGACC-3'); or $\beta$-actin (forward, 5'-CATGTACGTTGCTATCCAGGC-3' and reverse, 5'-CTCCTTAATGTCACGCACGA-3') under the following conditions: $10 \mathrm{~min}$ at $94^{\circ} \mathrm{C}$, followed by 40 cycles of $15 \mathrm{sec}$ at $95^{\circ} \mathrm{C}$ and $1 \mathrm{~min}$ at $60^{\circ} \mathrm{C}$. $\beta$-actin served as an internal control. The relative mRNA expression of MMP-3 and E-cadherin was determined by the $2^{-\Delta \Delta \mathrm{Ct}}$ method.

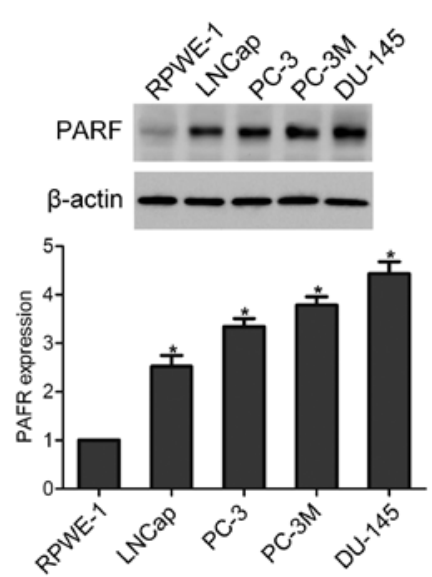

Figure 1. Expression of PAFR in human normal prostate RPWE-1 cells and prostate cancer LNCap, PC-3, PC-3M and DU-145 cells. " $\mathrm{P}<0.05$.

ELISA assay. After stimulation with or without PAF, the supernatants of DU-145 cells was collected and subjected to MMP-3 ELISA assay (Invitrogen), according to the manufacturer's instruction. To detect MMP-3 level in transplanted tumor tissues of mice, total proteins from transplanted tumor tissues of mice were isolated in RIPA buffer with protease inhibitor, and then MMP-3 ELISA kit (Invitrogen) was used to determine MMP-3 protein expression.

In vitro CCK-8 proliferation assay. Cell proliferation was determined by CCK-8 assay kit (Jingmei Biotech Co., Ltd., Shanghai, China). Briefly, cells were seeded at 800 cell/well in a 96-well plate, and incubated for $6 \mathrm{~h}$. Then, cells were stimulated with or without PAF for $72 \mathrm{~h}$. Next, $15 \mu \mathrm{l}$ of CCK-8 was added into the plate and cells were further incubated for $2 \mathrm{~h}$. Finally, optical density (OD) was observed using a microplate reader (Bio-Rad Laboratories) at $490 \mathrm{~nm}$.

In vivo proliferation and metastasis assay. Male BABL/c nude mice at four weeks old were purchased and maintained in the pathogen-free conditions under the approval of the Animal Care and Use Committee of Wenzhou Medical University. Sixteen mice were randomly divided into two groups ( $\mathrm{n}=8$ each group). Then shControl cells or shPAFR cells $\left(2 \times 10^{5}\right.$ cells) were subcutaneously injected at the back of the mice. Tumors were formed in one week. The length and width of tumors in mice were measured every week, and tumor volumes were estimated with the formula of $0.52 \mathrm{x}$ length $\mathrm{x}$ width ${ }^{2}$. Seven weeks later, the mice were sacrificed. Tumor tissues were lysed in RIPA buffer to further detect MMP-3 and E-cadherin expression. The livers were fixed in $4 \%$ paraformaldehyde, sectioned into slices and stained with H\&E. Then the number of micrometastasis in liver was counted under a microscope.

Statistical analysis. Experiments were performed three times, and data are presented as mean \pm standard deviation (SD). Statistical analysis was performed using SPSS version 17.0 software (SPSS, Inc., Chicago, IL, USA). The statistical significant difference was determined by the Student's t-test between two groups, or by non-parametric ANOVA among multiple groups. $\mathrm{P}<0.05$ was considered to be statistically significant. 

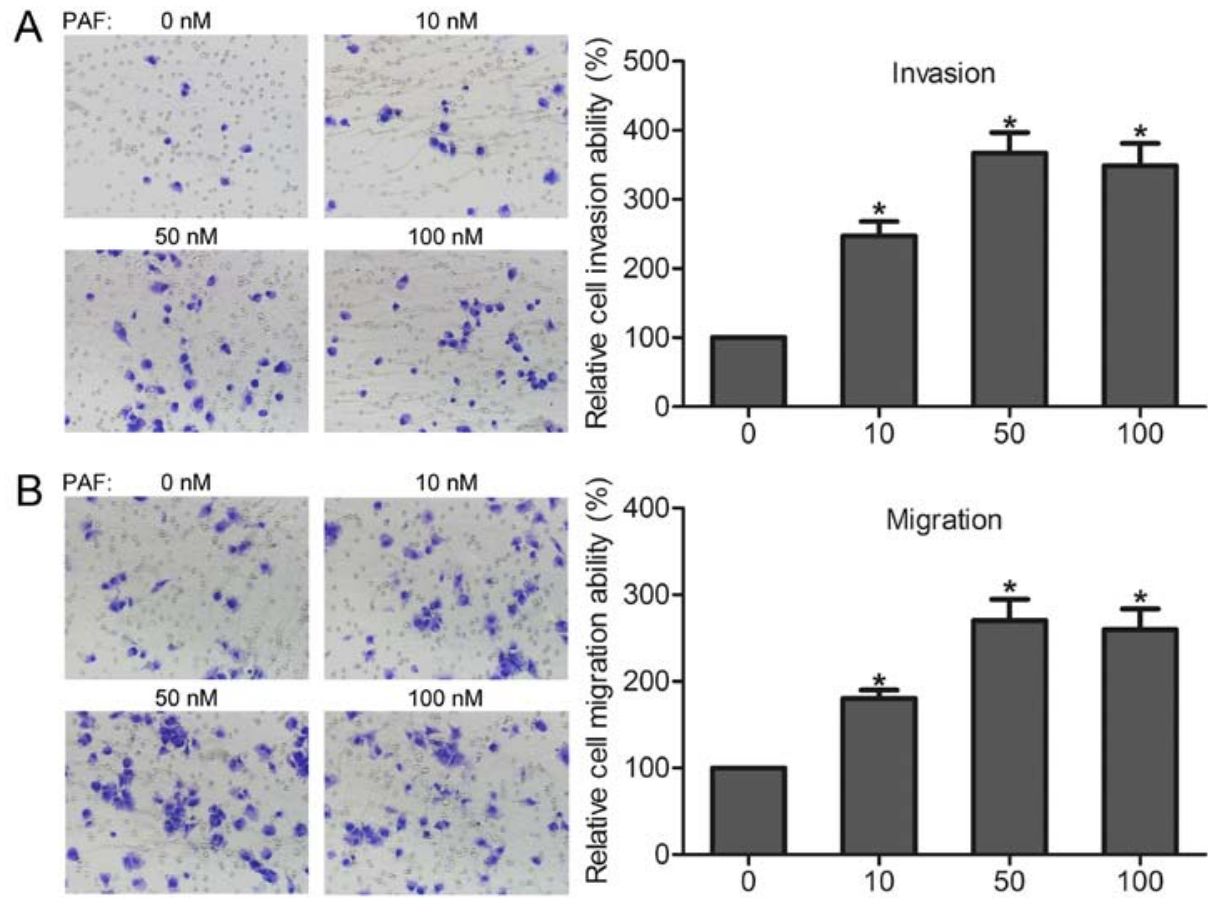

Figure 2. Effects of PAF stimulation (0-100 nM) on prostate cancer cell invasion and migration. (A) Invasion of the DU-145 cells induced by PAF stimulation was examined by in vitro invasion assay. (B) Migration of the DU-145 cells induced by PAF stimulation was examined by in vitro migration assay. "P $<0.05$.
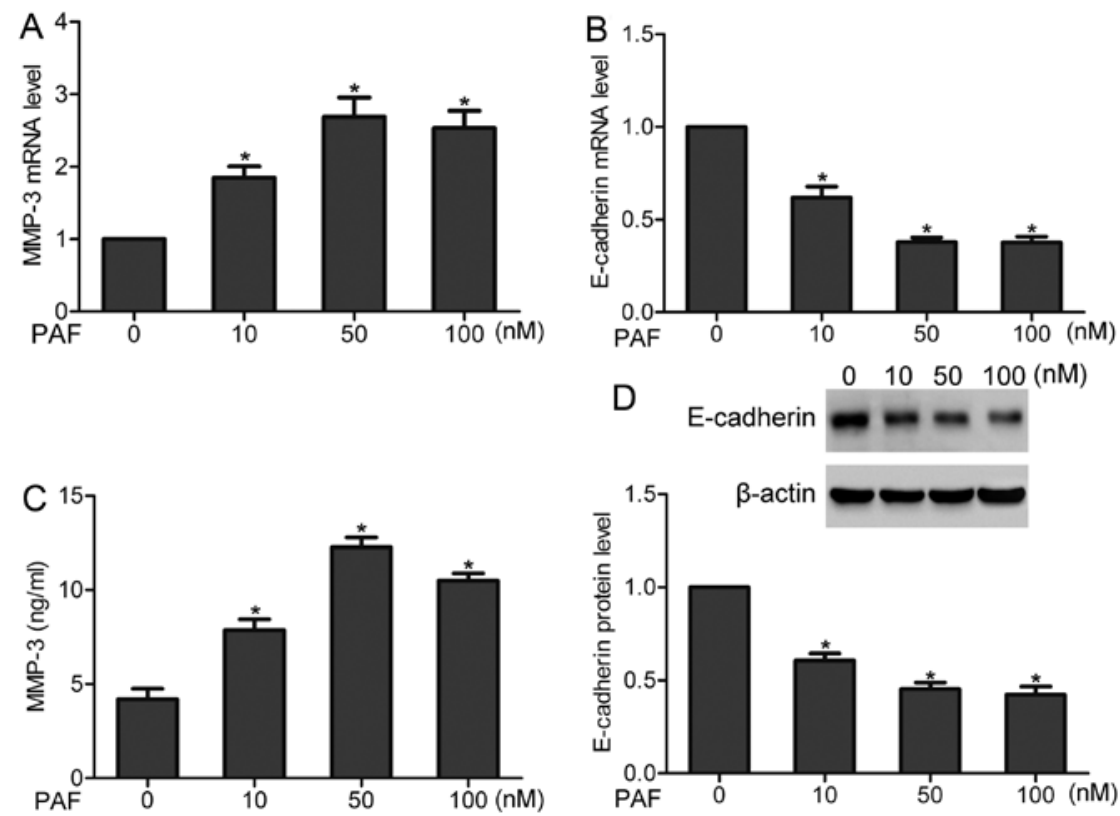

Figure 3. The expression of MMP-3 and E-cadherin after different concentrations of PAF stimulation (0-100 nM). (A) MMP-3 mRNA expression was detected by real-time PCR. (B) E-cadherin mRNA expression was detected by real-time PCR. (C) MMP-3 protein expression was detected by ELISA assay. (D) E-cadherin protein expression was detected by western blotting. ${ }^{*} \mathrm{P}<0.05$.

\section{Results}

Overexpression of PAFR in prostate cancer cells. In the present study we detected the protein level of PAFR in human normal prostate RPWE-1 cells and prostate cancer LNCap, PC-3, PC-3M and DU-145 cells by using western blotting. The results showed that the PAFR expression level was significantly higher in prostate cancer cells as compared to RPWE-1 cells (Fig. 1).
PAFR activation induces invasion and migration of prostate cancer cells in vitro. To investigate the role of PAFR in prostate cancer cells, DU-145 cells were selected and PAF was used to activate PAFR. In vitro invasion and migration assays were performed to determine the invasive and migration effect of PAFR activation on prostate cancer cells. We found that cell invasion was dose-dependently increased after incubation with different concentration of PAF (Fig. 2A). In vitro migration assay showed that PAF dose-dependently induced the 
A

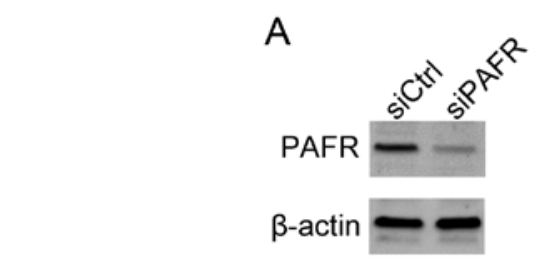

B
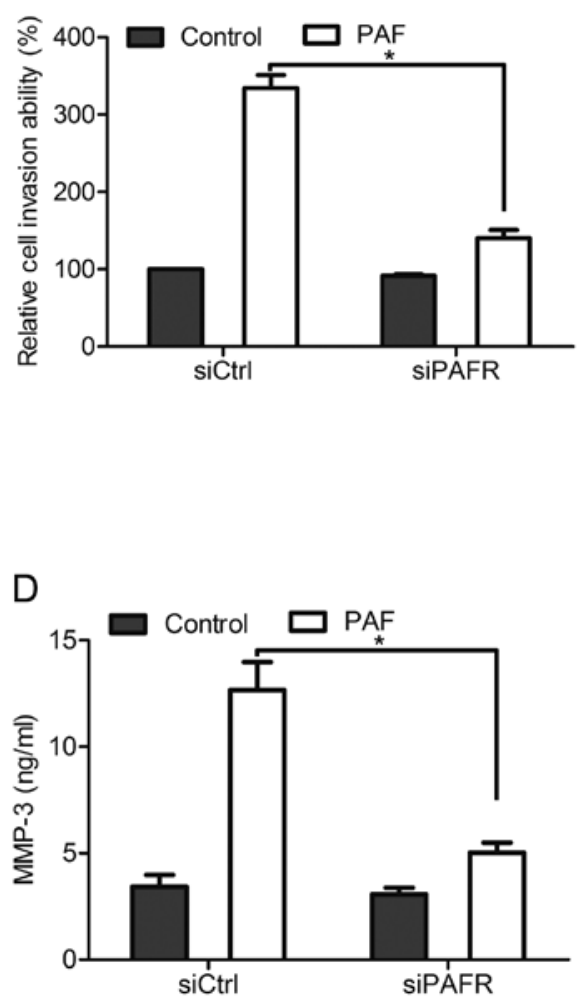
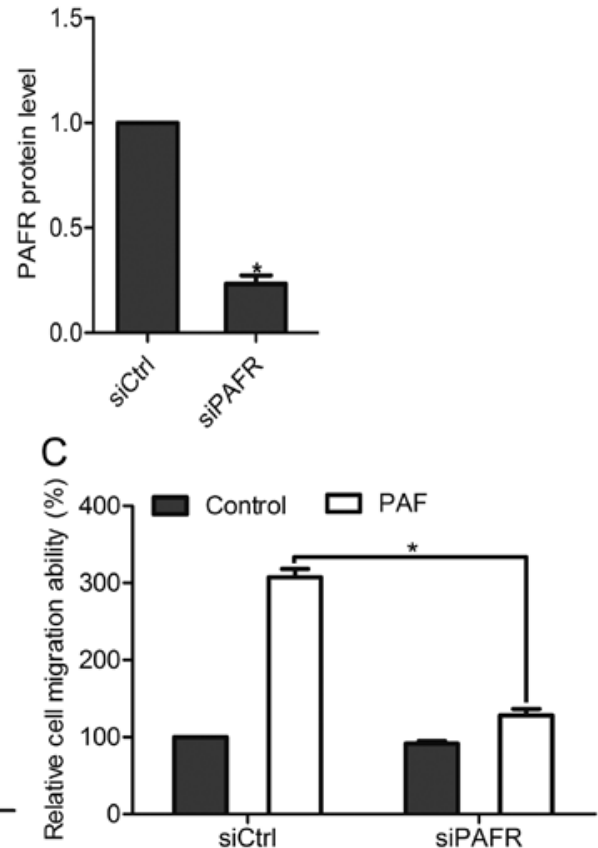

E

PAF $(50 \mathrm{nM}) \frac{\text { siCtrl }}{+} \frac{\text { siPAFR }}{+}$

E-cadherin $-\infty-$

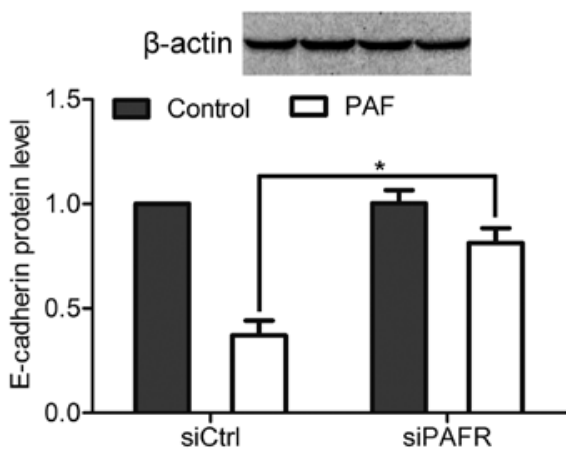

Figure 4. Effect of PAFR knockdown on PAF-mediated cell invasion, migration and expression of MMP-3 and E-cadherin. (A) Knockdown efficiency of siPAFR in DU-145 cells was detected by western blotting. Cells were transfected with siPAFR or siCtrl, and then were stimulated with PAF (50 nM) for $16 \mathrm{~h}$. (B) Effect of PAFR knockdown on PAF-mediated cell invasion was detected by in vitro invasion assay. (C) Effect of PAFR knockdown on PAF-mediated cell migration was detected by in vitro migration assay. (D) Effect of PAFR knockdown on PAF-mediated expression of MMP-3 was evaluated by ELISA assay. (E) Effect of PAFR knockdown on PAF-mediated expression of E-cadherin was detected by western blotting. "P<0.05.

migration of DU-145 cells (Fig. 2B). These findings indicate that PAFR activation is involved in prostate cancer cell invasion and migration in vitro.

PAFR activation regulates the expressions of $M M P-3$ and $E$-cadherin in vitro. After incubation of PAF with a concentration of 10,50 and $100 \mathrm{nM}$, respectively, the mRNA and protein levels of MMP-3 were observed by real-time PCR and ELISA assay, respectively. The results showed that the production of MMP-3 was increased after PAF stimulation, in a dose-dependent manner (Fig. 3A and C). We further found that PAF stimulation decreased the expression of E-cadherin at mRNA and protein levels (Fig. 3B and D). These findings suggest that PAFR activation can regulate the levels of MMP-3 and E-cadherin in prostate cancer cells.

Knockdown of PAFR inhibits prostate cancer cell invasion and migration in vitro. To confirm the effect of PAFR on prostate cancer cells, DU-145 cells were transfected with siPAFR to reduce PAFR expression. Using western blotting, we found that siPAFR significantly reduced PAFR expression by $>70 \%$ compared with the siCtrl transfection (Fig. 4A). Using in vitro invasion assay and migration assay, we found that PAF stimulation promoted the invasion and migration in siCtrl cells, whereas knockdown of PAFR attenuated the invasion and migration of DU-145 cells induced by PAF (Fig. 4B and C). Furthermore, after knockdown of PAFR, PAF-mediated increase of MMP-3 expression, as well as decrease of E-cadherin was suppressed (Fig. 4D and E). These data confirm that PAFR contributes to prostate cancer cell invasion and migration.

PAFR activation induces activation of ERK1/2 in vitro. Next, we found that PAF stimulation induced activation of ERK1/2 in DU-145 cells, in a time-dependent manner, and peak activation was observed at $30 \mathrm{~min}$ (Fig. 5A). However, knockdown of 
A
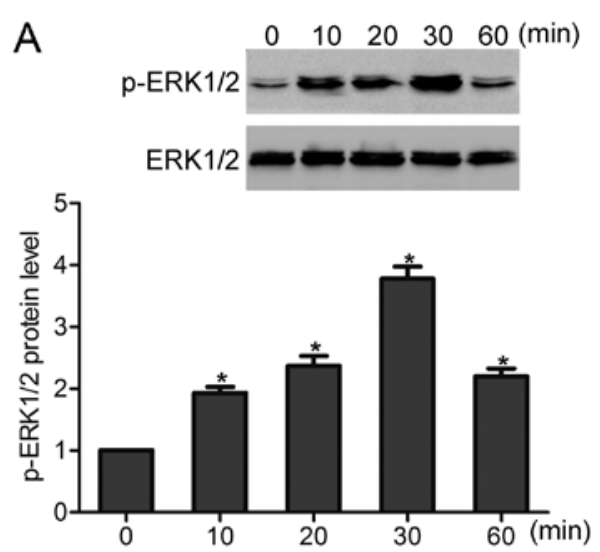

B

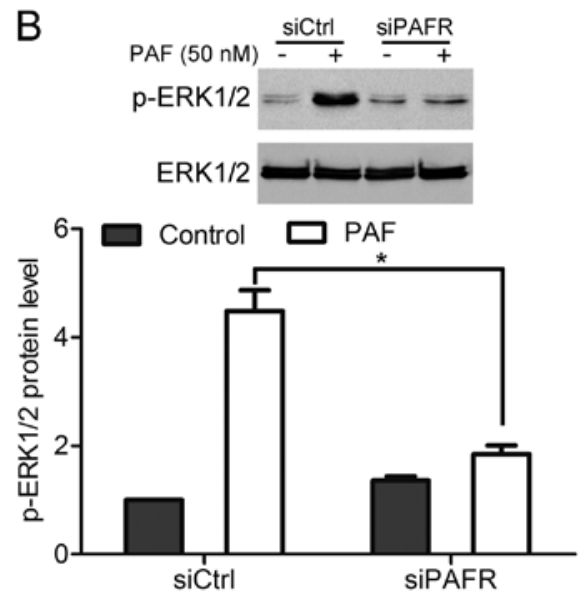

Figure 5. Involvement of PAFR activation in ERK1/2 activity. (A) Cells were stimulated with PAF (50 nM) for 0, 10, 20, 30 or 60 min, ERK1/2 activation was observed by western blotting. (B) Effect of PAFR knockdown on PAF-mediated ERK1/2 activation. ${ }^{*} \mathrm{P}<0.05$.
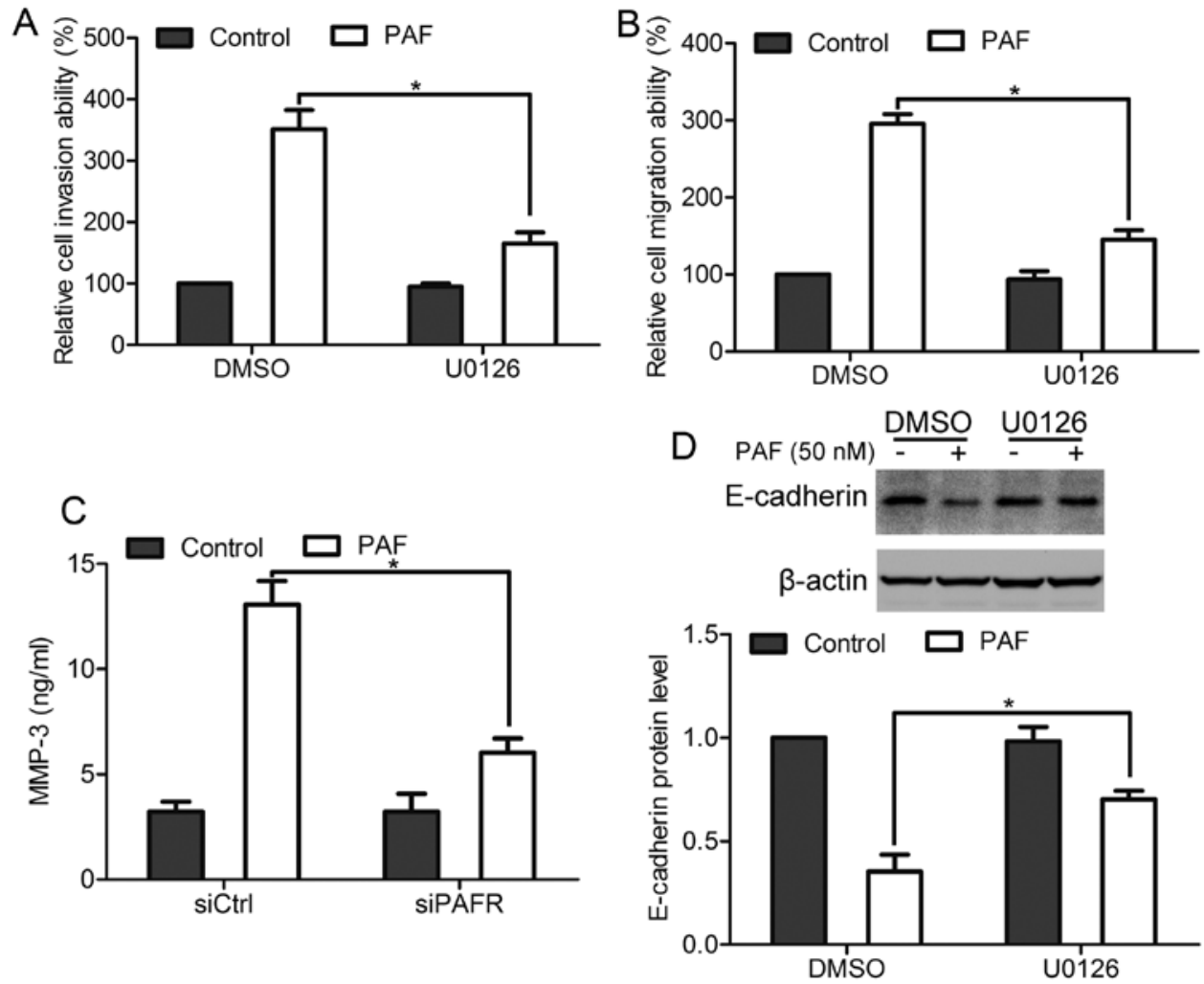

Figure 6. Role of ERK1/2 pathway in PAFR-mediated cell invasion, migration and expression of MMP-3 and E-cadherin. Cells were pretreated with U0126 $(10 \mathrm{nM})$ for $1 \mathrm{~h}$, and then were stimulated with PAF. (A) Effect of ERK1/2 activation on cell invasion was detected by in vitro invasion assay. (B) Effect of ERK1/2 activation on cell migration was detected by in vitro migration assay. (C) Effect of ERK1/2 activation on MMP-3 expression was evaluated by ELISA assay. (D) Effect of ERK1/2 activation on E-cadherin expression was detected by western blotting. "P<0.05.

PAFR inhibited PAF-induced activation of ERK1/2 (Fig. 5B). These findings suggest that PAF can mediate ERK1/2 activity via PAFR.

ERK1/2 pathway is required for PAFR-mediated prostate cancer cell invasion and migration. To investigate the function of ERK1/2 pathway in PAFR-mediated prostate cancer cell invasion and migration, cells were incubated with U0126 for $1 \mathrm{~h}$ before PAF stimulation to inhibit ERK1/2 activation. In vitro invasion assay and migration assay showed that inhi- bition of ERK1/2 activation suppressed PAF-mediated cell invasion and migration (Fig. 6A and B). Moreover, inhibition of ERK1/2 activation inhibited PAF-mediated increase of MMP-3 expression and decrease of E-cadherin expression (Fig. 6C and D). Together, these data suggest that ERK1/2 pathway is essential for PAFR-mediated prostate cancer cell invasion and migration.

PAFR activation promotes the metastasis of prostate cancer cells in vivo. To detect the effect of PAFR on the metastasis 
A

A
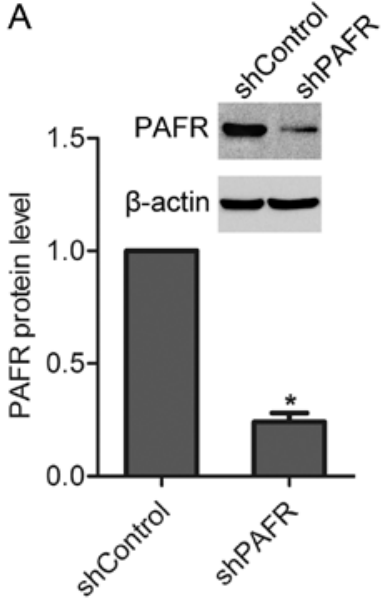

B shControl

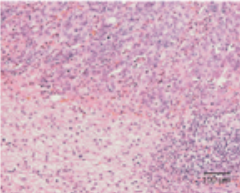

ShPAFR
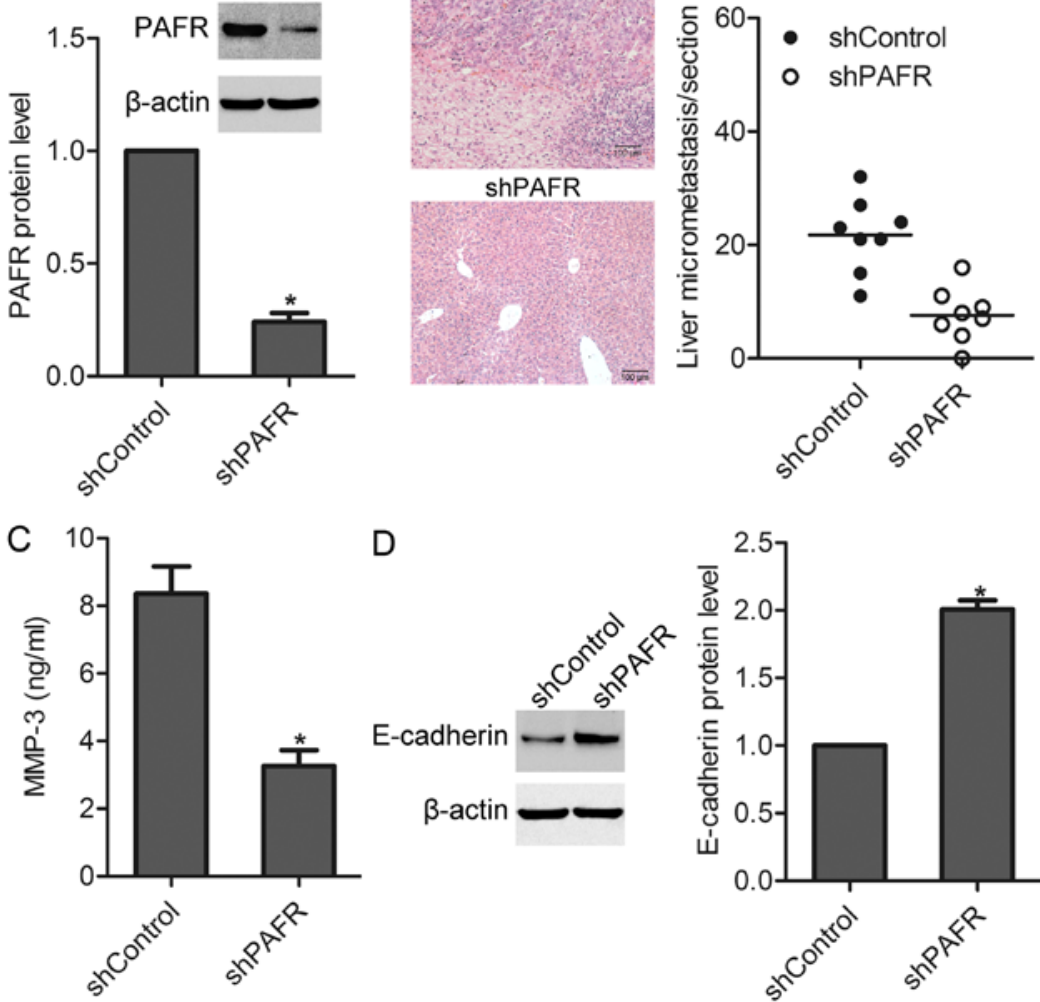

Figure 7. Effect of PAFR knockdown on prostate cancer cell metastasis, MMP-3 and E-cadherin expression in vivo. (A) Cells were stably transfected with pcDNA3.1-shPAFR, knockdown efficiency was detected by western blotting. (B) shControl and shPAFR cells were injected subcutaneously into two groups of nude mice $(n=8)$, respectively. Eight weeks later, the mice were sacrificed, and the livers were stained with H\&E. Liver micrometastasis was observed and counted under a microscope. (C) Expression of MMP-3 in tumor tissues was observed by ELISA assay. (D) Expression of E-cadherin in tumor tissues was observed by western blotting. ${ }^{*} \mathrm{P}<0.05$.
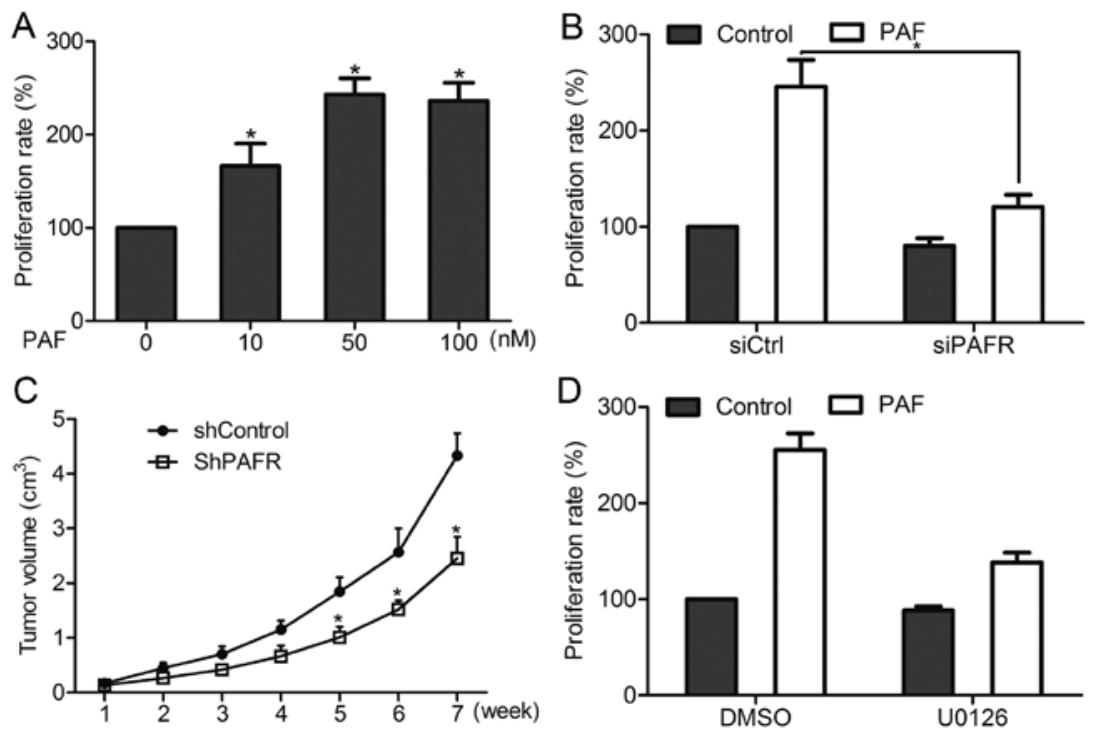

Figure 8. Effect of PAFR activation on cell proliferation in vitro and in vivo. (A) Cells were stimulated with different concentrations of PAF ( 0 -100 nM) for $72 \mathrm{~h}$. Cell proliferation was detected by in vitro CCK-8 proliferation assay. (B) Cells were silenced by siPAFR, and then were stimulated with 50 nM PAF for $72 \mathrm{~h}$. Cell proliferation was detected by in vitro CCK-8 proliferation assay. (C) shControl and shPAFR cells were injected subcutaneously into two groups of nude mice $(\mathrm{n}=8)$, respectively. Cell proliferation was examined by in vivo proliferation assay. (D) Cells were pretreated with U0126 (10 nM) for $1 \mathrm{~h}$, and then were stimulated with $50 \mathrm{nM}$ PAF for $72 \mathrm{~h}$. Cell proliferation was detected by in vitro CCK-8 proliferation assay. ${ }^{*} \mathrm{P}<0.05$.

of prostate cancer cells in vivo, cells were transfected with pcDNA3.1-shPAFR to stably decrease PAFR expression
(Fig. 7A). Then, the mice were injected subcutaneously with shControl and shPAFR cells into the back, respectively. Eight 
weeks later, the mice were sacrificed, and liver micrometastasis was observed under a microscope. The results showed that knockdown of PAFR greatly decreased the number of micrometastasis in the liver (Fig. 7B). We found that after knockdown of PAFR, E-cadherin expression was increased while MMP-3 expression was decreased in tumor tissues (Fig. 7C and D). These results suggest that PAFR participates in the metastasis of prostate cancer cells in vivo.

Effect of PAFR on prostate cancer cell proliferation in vitro and in vivo. We examined the effect of PAFR on cell proliferation of prostate cancer cells. In vitro $\mathrm{CCK}-8$ proliferation assay showed that PAF stimulation dose-dependently promoted the proliferation of DU-145 cells in vitro, whereas knockdown of PAFR suppressed the effect of PAF on cell proliferation (Fig. 8A and B). Similarly, in vivo growth assay showed that knockdown of PAFR inhibited the proliferation of DU-145 cells in mice (Fig. 8C). These results suggest that PAFR is involved in prostate cancer cell proliferation. Moreover, inhibition of ERK1/2 pathway attenuated PAF-mediated cell growth of DU-145 cells in vitro (Fig. 8D), indicating PAFR regulated prostate cancer cell growth via the ERK1/2 pathway.

\section{Discussion}

In the present study, we found that PAFR induced activation of the ERK1/2 pathway, leading to the upregulation of MMP-3 and downregulation of E-cadherin expression, ultimately inducing the invasion and metastasis of prostate cancer cells in vitro and in vivo. We also found that PAFR contributed to prostate cancer cell proliferation via ERK1/2 pathway. These findings suggest that PAFR may be an essential mediator in prostate cancer progression.

PAF plays an important role in many cellular processes. It is reported that PAF can induce activation of matrix metalloproteinase-2 activity and vascular endothelial cell invasion and migration (10). In cancer, Melnikova et al (11) have reported that PAF contributs to the metastasis of melanoma. PAF binds the PAFR, which belongs to GPCR family and is involved in the tumorigenesis and progression of many cancers. It is reported that PAFR is essential for the malignant potential in BRCA1 dysfunctional at-risk ovarian epithelium (12), and PAFR activation promotes the growth of ovarian cancer cells (13). In addition, studies have found that crosstalk between protease-activated receptor 1 and PAFR regulates melanoma metastasis (14) and PAFR activation augments the growth and metastasis of lung cancer (15). However, some studies have found that lower expression of PAFR correlats with poor differentiation and a poor prognosis in patients with hepatocellular carcinoma after hepatectomy (16), and PAFR expression is negative associated with histopathological stage and grade and patient survival in gastric adenocarcinoma (9). In prostate cancer cells, Jan and Chao (17) found that a specific PAFR antagonist inhibits prostate cancer cell growth, but the function of PAFR in the progression of prostate cancer cells and the underlying molecular mechanisms are still not very clear. In the present study, we found that PAFR expression was upregulated in prostate cancer cells. Activation of PAFR by PAF dose-dependently stimulated the growth, invasion and migration of prostate cancer cells in vitro. Knockdown of PAFR inhibited PAF-mediated cell growth, invasion and migration. Furthermore, knockdown of PAFR suppressed the growth and metastasis of prostate cancer cells in vivo. These data suggest that PAFR may act as a vital mediator in prostate cancer cells.

We further found that PAFR activation decreased E-cadherin expression and increased MMP-3 expression of prostate cancer cells in vitro, whereas knockdown of PAFR attenuated the effect of PAF on E-cadherin and MMP-3 expression. Moreover, knockdown of PAFR increased E-cadherin expression and decreased MMP-3 expression of prostate cancer cells in vivo. MMP-3 is an important member of the matrix metalloproteinase family, which is required for the dissolution of stromal collagen during tumor dissemination (18). It is considered that MMP-3 is involved in the invasion and metastasis of many cancers including prostate cancer (19-21). E-cadherin is a cell-cell adhesion protein and a well-documented tumor suppressor (22). Many studies have proved that downregulation of E-cadherin participates in the occurrence and development of prostate cancer $(23,24)$. In the present study, we showed that PAFR induced prostate cancer cell invasion and metastasis in vitro and in vivo, and decreased E-cadherin expression and increased MMP-3 expression, suggesting that PAFR may induce the invasion and metastasis of prostate cancer cells via regulation of MMP-3 and E-cadherin expression.

The MAPK ERK1/2 pathway is involved in a wide variety of cellular processes in cancer development $(25,26)$. Studies have shown that ERK1/2 activation participates in cell growth, invasion, malignant transformation and drug resistance of prostate cancer $(27,28)$. The ERK1/2 pathway can be activated by many growth factors and cytokines that are important in the progression of cancer. In the present study, we found that PAF induced activation of ERK1/2 via PAFR. Moreover, using an ERK1/2 specific inhibitor, we found that ERK1/2 pathway was required for PAFR-mediated cell growth, invasion and metastasis of prostate cancer cells.

In conclusion, our data demonstrate that PAFR is overexpressed in prostate cancer cells. PAFR promotes prostate cancer cell invasion and metastasis of prostate cancer cells in vitro and in vivo, possibly via activation of ERK1/2 pathway and regulation of E-cadherin and MMP-3 expression. We also showed that PAFR stimulates the growth of prostate cancer cells via ERK1/2 pathway. The present study provides evidence showing that PAFR may have a potential value in early detection and therapy for prostate cancer.

\section{References}

1. Siegel RL, Miller KD and Jemal A: Cancer statistics, 2015. CA Cancer J Clin 65: 5-29, 2015.

2. Cheville JC, Tindall D, Boelter C, Jenkins R, Lohse CM, Pankratz VS, Sebo TJ, Davis B and Blute ML: Metastatic prostate carcinoma to bone: Clinical and pathologic features associated with cancer-specific survival. Cancer 95: 1028-1036, 2002.

3. Zimmerman GA, McIntyre TM, Prescott SM and Stafforini DM: The platelet-activating factor signaling system and its regulators in syndromes of inflammation and thrombosis. Crit Care Med 30 (Suppl): S294-S301, 2002.

4. Melnikova V and Bar-Eli M: Inflammation and melanoma growth and metastasis: The role of platelet-activating factor (PAF) and its receptor. Cancer Metastasis Rev 26: 359-371, 2007. 
5. BussolatiB,Biancone L,Cassoni P, Russo S, Rola-PleszczynskiM, Montrucchio G and Camussi G: PAF produced by human breas cancer cells promotes migration and proliferation of tumor cells and neo-angiogenesis. Am J Pathol 157: 1713-1725, 2000.

6. Fallani A, Calorini L, Mannini A, Gabellieri S, Mugnai G and Ruggieri S: Platelet-activating factor (PAF) is the effector of IFN gamma-stimulated invasiveness and motility in a B16 melanoma line. Prostaglandins Other Lipid Mediat 81: 171-177, 2006.

7. Chen J, Lan T, Zhang W, Dong L, Kang N, Zhang S, Fu M, Liu B, Liu K, Zhang C, et al: Platelet-activating factor receptormediated PI3K/AKT activation contributes to the malignant development of esophageal squamous cell carcinoma. Oncogene 34: 5114-5127, 2015 .

8. Yu Y, Zhang M, Zhang X, Cai Q, Hong S, Jiang W and Xu C: Synergistic effects of combined platelet-activating factor receptor and epidermal growth factor receptor targeting in ovarian cancer cells. J Hematol Oncol 7: 39, 2014

9. Giaginis C, Kourou E, Giagini A, Goutas N, Patsouris E, Kouraklis G and Theocharis S: Platelet-activating factor (PAF) receptor expression is associated with histopathological stage and grade and patients' survival in gastric adenocarcinoma. Neoplasma 61: 309-317, 2014.

10. Axelrad TW, Deo DD, Ottino P, Van Kirk J, Bazan NG, Bazan HE and Hunt JD: Platelet-activating factor (PAF) induces activation of matrix metalloproteinase 2 activity and vascular endothelial cell invasion and migration. FASEB J 18: 568-570, 2004.

11. Melnikova VO, Mourad-Zeidan AA, Lev DC and Bar-Eli M: Platelet-activating factor mediates MMP-2 expression and activation via phosphorylation of cAMP-response element-binding protein and contributes to melanoma metastasis. J Biol Chem 281: 2911-2922, 2006.

12. Zhang L, Wang D, Jiang W, Edwards D, Qiu W, Barroilhet LM, Rho JH, Jin L, Seethappan V, Vitonis A, et al: Activated networking of platelet activating factor receptor and FAK/ STAT1 induces malignant potential in BRCA1-mutant at-risk ovarian epithelium. Reprod Biol Endocrinol 8: 74, 2010.

13. Yu Y, Zhang X, Hong S, Zhang M, Cai Q, Zhang M, Jiang W and $\mathrm{Xu} C$ : The expression of platelet-activating factor receptor modulates the cisplatin sensitivity of ovarian cancer cells: A novel target for combination therapy. Br J Cancer 111: 515-524, 2014.

14. Melnikova VO, Balasubramanian K, Villares GJ, Dobroff AS, Zigler M, Wang H, Petersson F, Price JE, Schroit A, Prieto VG, et al: Crosstalk between protease-activated receptor 1 and platelet-activating factor receptor regulates melanoma cell adhesion molecule (MCAM/MUC18) expression and melanoma metastasis. J Biol Chem 284: 28845-28855, 2009.

15. Hackler PC, Reuss S, Konger RL, Travers JB and Sahu RP: Systemic platelet-activating factor receptor activation augments experimental lung tumor growth and metastasis. Cancer Growth Metastasis 7: 27-32, 2014.
16. Kitagawa D, Taketomi A, Kayashima H, Kuroda Y, Itoh S, Yamashita Y and Maehara Y: Expression of platelet-activating factor receptor: A novel prognosticator in patients with hepatocellular carcinoma following hepatectomy. Oncology 72: 381-387, 2007.

17. Jan CR and Chao YY: Novel effect of Y-24180, a presumed specific platelet activation factor receptor antagonist, on $\mathrm{Ca}^{2+}$ levels and growth of human prostate cancer cells. Cell Signal 16: 959-965, 2004.

18. Littlepage LE, Sternlicht MD, Rougier N, Phillips J, Gallo E, Yu Y, Williams K, Brenot A, Gordon JI and Werb Z: Matrix metalloproteinases contribute distinct roles in neuroendocrine prostate carcinogenesis, metastasis, and angiogenesis progression. Cancer Res 70: 2224-2234, 2010.

19. Slavin S, Yeh CR, Da J, Yu S, Miyamoto H, Messing EM, Guancial E and Yeh S: Estrogen receptor $\alpha$ in cancer-associated fibroblasts suppresses prostate cancer invasion via modulation of thrombospondin 2 and matrix metalloproteinase 3. Carcinogenesis 35: 1301-1309, 2014.

20. Zhu F, Liu P, Li J and Zhang Y: Eotaxin-1 promotes prostate cancer cell invasion via activation of the CCR3-ERK pathway and upregulation of MMP-3 expression. Oncol Rep 31: 2049-2054, 2014.

21. Yamashita CM, Radisky DC, Aschner Y and Downey GP: The importance of matrix metalloproteinase-3 in respiratory disorders. Expert Rev Respir Med 8: 411-421, 2014.

22. Canel M, Serrels A, Frame MC and Brunton VG: E-cadherinintegrin crosstalk in cancer invasion and metastasis. J Cell Sci 126: 393-401, 2013.

23. Davies G, Jiang WG and Mason MD: E-cadherin and associated molecules in the invasion and progression of prostate cancer. Oncol Rep 5: 1567-1576, 1998.

24. Khamis ZI, Iczkowski KA and Sang QX: Metastasis suppressors in human benign prostate, intraepithelial neoplasia, and invasive cancer: Their prospects as therapeutic agents. Med Res Rev 32: 1026-1077, 2012

25. Samatar AA and Poulikakos PI: Targeting RAS-ERK signalling in cancer: Promises and challenges. Nat Rev Drug Discov 13: 928-942, 2014

26. De Luca A, Maiello MR, D'Alessio A, Pergameno $M$ and Normanno N: The RAS/RAF/MEK/ERK and the PI3K/AKT signalling pathways: Role in cancer pathogenesis and implications for therapeutic approaches. Expert Opin Ther Targets 16 (Suppl 2): S17-S27, 2012.

27. McCubrey JA, Steelman LS, Chappell WH, Abrams SL, Wong EW, Chang F, Lehmann B, Terrian DM, Milella M, Tafuri A, et al: Roles of the Raf/MEK/ERK pathway in cell growth, malignant transformation and drug resistance. Biochim Biophys Acta 1773: 1263-1284, 2007.

28. Sosa MS, Avivar-Valderas A, Bragado P, Wen HC and AguirreGhiso JA: ERK $1 / 2$ and $\mathrm{p} 38 \alpha / \beta$ signaling in tumor cell quiescence: opportunities to control dormant residual disease. Clin Cancer Res 17: 5850-5857, 2011. 